Pacific Journal of Mathematics

THE SPACING OF THE MINIMA IN CERTAIN CUBIC 


\title{
THE SPACING OF THE MINIMA IN CERTAIN CUBIC LATTICES
}

\author{
H. C. Williams
}

Let $\mathscr{K}$ be a cubic field with negative discriminant; let $\mu, \nu \in \mathscr{K}$; and let $\mathscr{R}$ be a lattice with basis $\{1, \mu, \nu\}$ such that 1 is a minimum of $\mathscr{R}$. If

$$
1=\theta_{1}, \theta_{2}, \theta_{3}, \ldots, \theta_{n}, \ldots
$$

is a chain of adjacent minima of $\mathscr{R}$ with $\theta_{i+1}>\theta_{i}(i=1,2,3, \ldots)$, then

$$
\theta_{n+5} \geq \theta_{n+3}+\theta_{n} \text {. }
$$

This result can be used to prove that if $p$ is the period of Voronoi's continued fraction algorithm for finding the fundamental unit $\varepsilon_{0}$ of $\mathscr{K}$, then

$$
\varepsilon_{0}>\tau^{p / 2},
$$

where $\tau=(1+\sqrt{5}) / 2$. It is also shown that

$$
\theta_{n}>4^{[(n-1) / 7]} \text {. }
$$

1. Introduction. In order to discuss the problems considered in this paper, it is necessary to give a brief description of the properties of cubic lattices. For a more extensive and more general treatment of these topics we refer the reader to Delone and Faddeev [1].

Let $f(x) \in \mathbf{Z}[x]$ be a cubic polynomial, irreducible over the rationals 2 and having a negative discriminant. Let $\delta$ be the real zero of $f(x)$ and denote by $\mathscr{K}=\mathscr{Q}(\delta)$ the complex cubic field formed by adjoining $\delta$ to $\mathscr{Q}$. If $\mathscr{E}_{3}$ denotes Euclidean 3-space, we can associate with each $\alpha \in \mathscr{K}$ a point $A \in \mathscr{E}_{3}$, where

$$
A=\left(\alpha,\left(\alpha^{\prime}-\alpha^{\prime \prime}\right) / 2 i,\left(\alpha^{\prime}+\alpha^{\prime \prime}\right) / 2\right),
$$

$i^{2}+1=0$, and $\alpha^{\prime}, \alpha^{\prime \prime}$ are the conjugates of $\alpha$. Since $f(x)$ has a negative discriminant, all three components of $A$ must be real. If $\lambda, \mu, \nu \in \mathscr{K}$ and $\lambda, \mu, \nu$ are rationally independent, we define the cubic lattice $\mathscr{L}$ by

$$
\mathscr{L}=\left\{u \Lambda+v M+w N \mid(u, v, w) \in \mathbf{Z}^{3}\right\} .
$$

We say that $\mathscr{L}$ has a basis $\{\lambda, \mu, \nu\}$ and denote $\mathscr{L}$ by $\langle\lambda, \mu, \nu\rangle$. For the sake of convenience we will often use the expression $\alpha \in \mathscr{L}$ to denote that it is the corresponding point $A \in \mathscr{E}_{3}$ that is actually in $\mathscr{L}$. Also, if $\mathscr{L}=\langle\lambda, \mu, \nu\rangle$, we define $\alpha \mathscr{L}(\alpha \in \mathscr{K})$ to be the lattice $\langle\alpha \lambda, \alpha \mu, \alpha \nu\rangle$. 
If $A$ is any point of $\mathscr{L}$, we define the normed body of $A$ to be

$$
\begin{aligned}
\mathscr{N}(A) & =\mathscr{N}(\alpha) \\
& =\left\{\left.(x, y, z)\left|(x, y, z) \in \mathscr{E}_{3},\right| x|<| \alpha\left|, y^{2}+z^{2} \leq\right| \alpha^{\prime}\right|^{2}\right\} .
\end{aligned}
$$

This is a semi-open right circular cylinder, symmetric about the origin $O$ of $\mathscr{E}_{3}$, with axis the $x$-axis of $\mathscr{E}_{3}$. It should be mentioned at this point that if $\alpha, \beta \in \mathscr{K}$ and $\left|\alpha^{\prime}\right|=\left|\beta^{\prime}\right|$, then $\alpha= \pm \beta$ (see [1], p. 274). Thus, if $\left|\beta^{\prime}\right|=\left|\alpha^{\prime}\right|$, then $B \notin \mathscr{N}(\alpha)$.

We say that $\phi(\neq 0) \in \mathscr{K}$ or the point $\Phi$ corresponding to $\phi$ is a minimum of $\mathscr{L}$ if $\mathscr{N}(\phi) \cap \mathscr{L}=\{0\}$. If $\psi$ and $\phi$ are minima of $\mathscr{L}$ and $\psi>\phi$, we say that $\psi$ and $\phi$ are adjacent minima when there does not exist a non-zero $\chi \in \mathscr{L}$ such that

$$
\phi<\chi<\psi \text { and }\left|\chi^{\prime}\right|<\left|\phi^{\prime}\right| \text {. }
$$

If

$$
\theta_{1}, \theta_{2}, \theta_{3}, \ldots, \theta_{n}, \cdots
$$

is a sequence of minima of $\mathscr{L}$ such that $\theta_{i+1}>\theta_{i}$ and $\theta_{i+1}, \theta_{i}$ are adjacent $(i=1,2,3, \ldots, n, \ldots)$, we call (1.1) a chain of minima of $\mathscr{L}$. By using Minkowski's theorem (see [1]) we can prove that such chains always exist in $\mathscr{L}$.

If $\mathscr{R}=\langle 1, \mu, \nu\rangle$ and 1 is a minimum of $\mathscr{R}$, we say that $\mathscr{R}$ is a reduced lattice. In this paper we shall be concerned with the problem of how closely spaced the minima of $\mathscr{R}$ can be. We will show that if $\theta_{1}=1$ and $\theta_{4}<\theta_{2}+1$, then $\theta_{2}+\theta_{3}=\theta_{4}+1$. We can use this result to prove that if $\varepsilon_{0}$ is the fundamental unit of $\mathscr{K}$, then

$$
\varepsilon_{0}>\tau^{p / 2}
$$

where $p$ is the period of Voronoi's continued fraction algorithm for finding $\varepsilon_{0}$ and $\tau=(1+\sqrt{5}) / 2$. We will also show that $\theta_{5} \geq \theta_{3}+1>2$ and $\theta_{8}>4$. The methods used to prove these results are completely elementary.

2. Preliminary results. From [1] or Williams and Dueck [3] we see that if $\mathscr{R}_{1}=\mathscr{R}$ (a reduced lattice), $\theta_{g}^{(m)}$ is the minimum of $\mathscr{R}_{m}$ adjacent to 1 and $\mathscr{R}_{m+1}$ is defined to be $\left(1 / \theta_{g}^{(m)}\right) \mathscr{R}_{m}$, then $\theta_{n} \mathscr{R}_{n}=\mathscr{R}_{1}$, where $\mathscr{R}_{n}$ is a reduced lattice and

$$
\theta_{n}=\prod_{i=1}^{n-1} \theta_{g}^{(i)}
$$


We shall need to make use of these results together with several others established in [3]; however, we first give some simple lemmas concerning points of $\mathscr{R}$. Throughout this work we will use $\theta$ to denote the minimum of $\mathscr{R}$ adjacent to $1, \omega$ to denote the minimum of $\mathscr{R}$ adjacent to $\theta$, and $\chi$ to denote the minimum of $\mathscr{R}$ adjacent to $\omega$. That is, $\theta=\theta_{2}, \omega=\theta_{3}$, $\chi=\theta_{4}$. Note that if $\gamma \in \mathscr{R},|\gamma|<\theta$, and $\left|\gamma^{\prime}\right| \leq 1$, we must have $\gamma=0$ or $\gamma= \pm 1$. We also have

LEMMA 2.1. If $\alpha \in \mathscr{R}$ and $0<\alpha<\theta+1$, then either $\alpha=1,2$ or $\left|\alpha^{\prime}-1\right|>1$. Further, if $\alpha, \beta \in \mathscr{R}, \alpha \neq \beta$, and $\theta<\alpha, \beta<\theta+1$, then $\left|\alpha^{\prime}-\beta^{\prime}\right|>1$.

Proof. We have $-1<\alpha-1<\theta$; thus, if $\left|\alpha^{\prime}-1\right| \leq 1$, we get $\alpha-1$ $=0$, . Since $\theta<\alpha, \beta<\theta+1$, we have $|\alpha-\beta|<1$. It follows that if $\left|\alpha^{\prime}-\beta^{\prime}\right|<1$, then $\alpha=\beta$. If $\left|\alpha^{\prime}-\beta^{\prime}\right|=1$, then $\alpha=\beta \pm 1$, which is also impossible.

From this result we see that $\left|\theta^{\prime}-1\right|>1$ and if $x<\theta+1$, then $\left|\omega^{\prime}-1\right|>1$ and $\left|\chi^{\prime}-1\right|>1$.

In order to develop further results we define

$$
\eta_{\alpha}=\left(\alpha^{\prime}-\alpha^{\prime \prime}\right) / 2 i, \quad \zeta_{\alpha}=\left(\alpha^{\prime}+\alpha^{\prime \prime}\right) / 2
$$

for any $\alpha \in \mathscr{K}$. Note that

$$
\left|\alpha^{\prime}\right|^{2}=\left|\alpha^{\prime \prime}\right|^{2}=\alpha^{\prime} \alpha^{\prime \prime}=\eta_{\alpha}^{2}+\zeta_{\alpha}^{2} .
$$

Also, if $\alpha \in \mathscr{R}$ and $\eta_{\alpha} \in \mathscr{2}$, then $\alpha \in \mathbf{Z}$ and $\eta_{\alpha}=0$ (see [3]). Hence, $\eta_{\alpha} \neq 0$ if $\alpha=\theta_{i}(i>1)$.

LEMMA 2.2. If $\alpha, \beta \in \mathscr{R},\left|\alpha^{\prime}\right|<1,\left|\beta^{\prime}\right|<1,\left|\alpha^{\prime}-1\right|>1, \mid \beta^{\prime}-\alpha^{\prime}+$ $1 \mid>1$, then $\left|\beta^{\prime}-\alpha^{\prime}+2\right|>1$.

Proof. Since $\left|\beta^{\prime}\right|<1$, we have $\zeta_{\beta}>-1$ by (2.3). Further, since $\left|\alpha^{\prime}\right|<1$ and $\left|\alpha^{\prime}-1\right|>1$, we must have $\zeta_{\alpha}<1 / 2$; thus, $\zeta_{\beta}-\zeta_{\alpha}+1>-1 / 2$ and

$$
\left|\beta^{\prime}-\alpha^{\prime}+2\right|^{2}=\left|\beta^{\prime}-\alpha^{\prime}+1\right|^{2}+2\left(\zeta_{\beta}-\zeta_{\alpha}+1\right)+1>1 .
$$

LEMmA 2.3. If $\alpha, \beta \in \mathscr{R},\left|\alpha^{\prime}-1\right|>1,\left|\alpha^{\prime}+1\right|>1,\left|\alpha^{\prime}\right|<1,\left|\beta^{\prime}\right|<1$, $\eta_{\beta} \eta_{\alpha}>0$, and $\left|\beta^{\prime}-\alpha^{\prime}\right|>1$, then $\left|\eta_{\alpha}\right|>\left|\eta_{\beta}\right|$.

Proof. Suppose $\left|\eta_{\alpha}\right| \leq\left|\eta_{\beta}\right|$ and consider Figure 1 . 


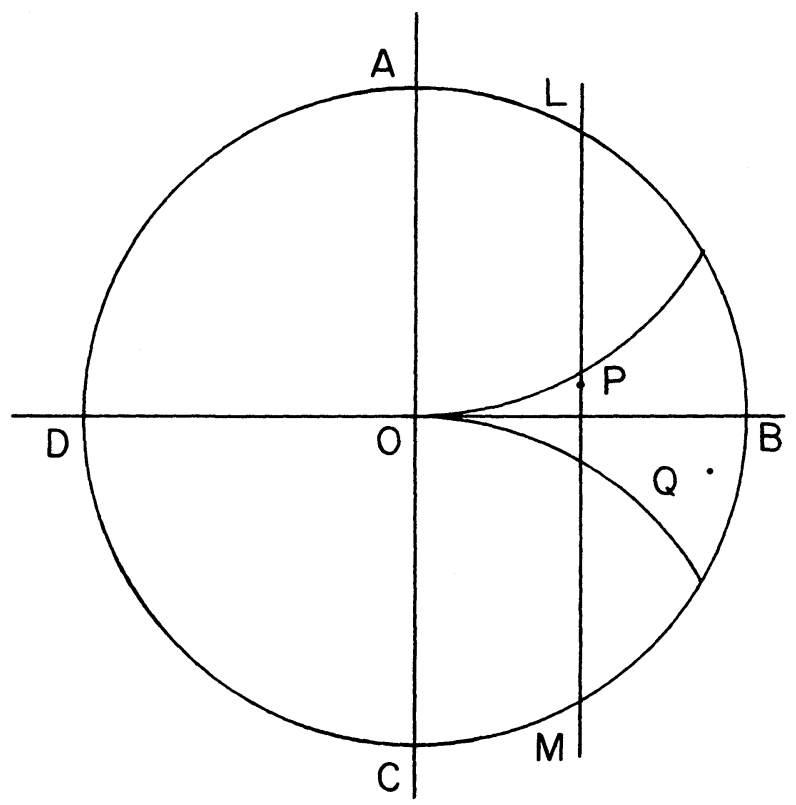

Figure 1

Here $P=\left(\left|\eta_{\alpha}\right|, \zeta_{\alpha}\right), Q=\left(\left|\eta_{\beta}\right|, \zeta_{\beta}\right)$. Let the chord through $P$ parallel to $A C$ meet the circle $A B C D$ (radius 1 , centre $O$ ) at $L$ and $M$. Since $\left|\alpha^{\prime}+1\right|>1$, we have $P L<1$; also, since $\left|\alpha^{\prime}-1\right|>1$, we have $P M<1$. Since $\overline{P Q}<$ $\max (\overline{P L}, \overline{P M})$, we get $\overline{P Q}=\left|\beta^{\prime}-\alpha^{\prime}\right|<1$, a contradiction.

In the next sequence of lemmas we prove a number of results concerning points $\alpha \in \mathscr{R}$ such that $\left|\alpha^{\prime}\right|<1$. We first define $\kappa(\alpha)$ for $\alpha \in \mathscr{R}$ by

$$
\begin{aligned}
\kappa(\alpha) & =\left(\zeta_{\alpha}-1 / 2\right)^{2}+\left(\sqrt{3} / 2-\left|\eta_{\alpha}\right|\right)^{2} \\
& =\zeta_{\alpha}^{2}-\zeta_{\alpha}+\eta_{\alpha}^{2}-\sqrt{3}\left|\eta_{\alpha}\right|+1
\end{aligned}
$$

LEMMA 2.4. If $\alpha \in \mathscr{R},\left|\alpha^{\prime}\right|<1$, and $\kappa(\alpha) \geq 1$, then $\zeta_{\alpha} \leq 0,\left|\eta_{\alpha}\right|$ $\leq \sqrt{3} / 2$, and $\left|\zeta_{\alpha}\right| \geq\left|\eta_{\alpha}\right| / \sqrt{3}$.

Proof. Since $\left|\alpha^{\prime}\right|<1$, we have $\left|\eta_{\alpha}\right|<1$ and $\left|\zeta_{\alpha}\right|<1$; thus,

$$
-\sqrt{3} / 2<\sqrt{3} / 2-1<\sqrt{3} / 2-\left|\eta_{\alpha}\right|<\sqrt{3} / 2,
$$

and $\left(\zeta_{\alpha}-1 / 2\right)^{2} \geq 1 / 4$ by (2.4). If $0<\zeta_{\alpha}<1$, this latter result is not possible; hence, $\zeta_{\alpha} \leq 0$. If $\left|\eta_{\alpha}\right|>\sqrt{3} / 2$, then $\left|\zeta_{\alpha}\right|<1 / 2$ by (2.3) and the fact that $\left|\alpha^{\prime}\right|<1$; thus, by (2.4)

$$
\kappa(\alpha)<-1 / 2+\zeta_{\alpha}^{2}+\eta_{\alpha}^{2}-\zeta_{\alpha}<1 / 2-\zeta_{\alpha}<1,
$$


which is also not possible. Since $\left|\eta_{\alpha}\right|<\sqrt{3} / 2$, we have $\left|\eta_{\alpha}\right|<$ $3(\sqrt{3}-1 / \sqrt{3}) / 4$ and

$$
\left(\left|\eta_{\alpha}\right| / \sqrt{3}+1 / 2\right)^{2}+\left(\sqrt{3} / 2-\left|\eta_{\alpha}\right|\right)^{2} \leq 1
$$

It follows that since $\kappa(\alpha) \geq 1$, we must have $\left|\zeta_{\alpha}\right| \geq\left|\eta_{\alpha}\right| / \sqrt{3}$ by (2.4).

Corollary 2.4.1. If $\alpha \in \mathscr{R},\left|\alpha^{\prime}\right|<1$, and $\kappa(\alpha) \geq 1$, then $\left|\alpha^{\prime}+1\right| \leq 1$.

Proof. By the lemma, $1-\left|\eta_{\alpha}\right| / \sqrt{3}>0$ and $0<\zeta_{\alpha}+1 \leq$ $1-\left|\eta_{\alpha}\right| / \sqrt{3}$. Thus,

$$
\left|\alpha^{\prime}+1\right|^{2}=\left(\zeta_{\alpha}+1\right)^{2}+\eta_{\alpha}^{2} \leq\left(1-\left|\eta_{\alpha}\right| / \sqrt{3}\right)^{2}+\eta_{\alpha}^{2} \leq 1
$$

as $\left|\eta_{\alpha}\right|<\sqrt{3} / 2$.

LEMMA 2.5. If $\alpha, \beta \in \mathscr{R},\left|\alpha^{\prime}\right|<1,\left|\beta^{\prime}\right|<1,\left|\alpha^{\prime}-1\right|>1, \eta_{\alpha} \eta_{\beta}>0$, $\kappa(\alpha)<1$, and $\left|\alpha^{\prime}-\beta^{\prime}\right|>1$, then $\kappa(\beta)>1$.

Proof. The point $\left(\eta_{\alpha}, \zeta_{\alpha}\right)$ must lie in the Reuleaux triangle (see [3]) with vertices $O$ (the origin), $(\sigma \sqrt{3} / 2,1 / 2),(\sigma \sqrt{3} / 2,-1 / 2)$, where $\sigma=$ $\operatorname{sgn}\left(\eta_{\alpha}\right)$. If $\kappa(\beta) \leq 1$, then $\left(\eta_{\beta}, \zeta_{\beta}\right)$ is in the same Reuleaux triangle as $\left(\eta_{\alpha}, \zeta_{\alpha}\right)$; hence, $\left|\alpha^{\prime}-\beta^{\prime}\right| \leq 1$, which is impossible.

LEMMA 2.6. If $\alpha, \beta \in \mathscr{R},\left|\alpha^{\prime}\right|<1,\left|\beta^{\prime}\right|<1,\left|\alpha^{\prime}-1\right|>1,\left|\alpha^{\prime}+1\right|>1$, and $\kappa(\beta) \geq 1$, then $\left|1-\alpha^{\prime}-\beta^{\prime}\right|>1$.

Proof. Since $\left|\alpha^{\prime}\right|<1,\left|\alpha^{\prime}+1\right|>1$, and $\left|\alpha^{\prime}-1\right|>1$, we have $\left|\zeta_{\alpha}\right|<$ $1 / 2$ and $1-2 \zeta_{\alpha}>0$. Since $\left|\alpha^{\prime}-1\right|>1$ and $\kappa(\beta) \geq 1$, we also have (2.5)

$$
\begin{aligned}
\mid 1-\alpha^{\prime} & -\left.\beta^{\prime}\right|^{2} \\
& =1+\zeta_{\beta}^{2}-2 \zeta_{\beta}+\eta_{\beta}^{2}+2 \zeta_{\alpha} \zeta_{\beta}+2 \eta_{\alpha} \eta_{\beta}+\zeta_{\alpha}^{2}-2 \zeta_{\alpha}+\eta_{\alpha}^{2} \\
& >1+\zeta_{\beta}\left(-1+2 \zeta_{\alpha}\right)+2 \eta_{\alpha} \eta_{\beta}+\sqrt{3}\left|\eta_{\beta}\right|
\end{aligned}
$$

by (2.4) and the fact that $\left|\alpha^{\prime}-1\right|>1$. By Lemma 2.4, we have $\zeta_{\alpha} \leq 0$; hence, if $\eta_{\alpha} \eta_{\beta} \geq 0$, we get $\left|1-\alpha^{\prime}-\beta^{\prime}\right|>1$. If $\eta_{\alpha} \eta_{\beta}<0$, then from (2.5) and Lemma 2.4 we get

$$
\left|1-\alpha^{\prime}-\beta^{\prime}\right|^{2}>1+\left|\eta_{\beta}\right|\left(\left(1-2 \zeta_{\alpha}\right) / \sqrt{3}+\sqrt{3}-2\left|\eta_{\alpha}\right|\right) \text {. }
$$

Since $\zeta_{\alpha}<\sqrt{1-\eta_{\alpha}^{2}}$, we have

$$
\left(1-2 \zeta_{\alpha}\right) / \sqrt{3}+\sqrt{3}-2\left|\eta_{\alpha}\right|>\left(1-2 \sqrt{1-\eta_{\alpha}^{2}}\right) / \sqrt{3}+\sqrt{3}-2\left|\eta_{\alpha}\right|
$$


But $2 / \sqrt{3}>1>\left|\eta_{\alpha}\right|$ and $\sqrt{1-\eta_{\alpha}^{2}} / \sqrt{3}<2 / \sqrt{3}-\left|\eta_{\alpha}\right|$; hence,

$$
\left(1-2 \sqrt{1-\eta_{\alpha}^{2}}\right) / \sqrt{3}+\sqrt{3}-2\left|\eta_{\alpha}\right|>0 \text {. }
$$

Corollary 2.6.1. If $\alpha, \beta \in \mathscr{R},\left|\alpha^{\prime}\right|<1, \quad\left|\beta^{\prime}\right|<1, \quad\left|\alpha^{\prime}-1\right|>1$, $\left|\alpha^{\prime}+1\right|>1,\left|\beta^{\prime}-\alpha^{\prime}\right|>1$, and $\left|\beta^{\prime}-\alpha^{\prime}+1\right|<1$, then $\kappa(\gamma)<1$, where $\gamma=\beta-\alpha+1$.

Proof. We have $\left|\gamma^{\prime}\right|<1$; thus, if $\kappa(\gamma) \geq 1$, then $\left|1-\alpha^{\prime}-\gamma^{\prime}\right|=\left|\beta^{\prime}\right|$ $>1$, which is not so.

We will also require some lemmas whose proofs have already appeared in [3]. We will only give the statements of these results here; however, we mention that the proofs of these lemmas are elementary and require, for the most part, only results from simple plane geometry.

LEMMA 2.7 (Lemma 6.1 of [3]). If $\alpha, \beta \in \mathscr{R},\left|\alpha^{\prime}\right|<1,\left|\beta^{\prime}\right|<1$, and $2 \alpha=\beta+1$, then $\left|\alpha^{\prime}-1\right| \leq 1$.

LeMMA 2.8 (Lemma 5.4 of [3]). If $\alpha, \beta \in \mathscr{R},\left|\alpha^{\prime}\right|<1,\left|\beta^{\prime}\right|<1$, $\left|\alpha^{\prime}-1\right|>1,\left|\beta^{\prime}-1\right|>1, \eta_{\alpha} \eta_{\beta}>0,\left|\alpha^{\prime}-\beta^{\prime}\right|>1$, and $\left|\alpha^{\prime}+1\right|>1(<$ $1)$, then $\left|\beta^{\prime}+1\right|<1(>1)$.

LeMma 2.9 (Lemma 6.2 of [3]). Let $\alpha, \beta, \gamma \in \mathscr{R}$, where $\alpha, \beta, \gamma$ are distinct, $\left|\alpha^{\prime}\right|<1,\left|\beta^{\prime}\right|<1,\left|\gamma^{\prime}\right|<1$, and $\left|\alpha^{\prime}-1\right|>1,\left|\beta^{\prime}-1\right|>1$, $\mid \gamma^{\prime}-$ $1 \mid>1$. If $\eta_{\alpha} \eta_{\beta}>0$ and $\eta_{\beta} \eta_{\alpha}>0$, there cannot exist any $b$ such that

$$
b \leq \alpha, \beta, \gamma<b+1 \text {. }
$$

LEMMA 2.10 (Lemma 6.3 of [3]). Let $\alpha, \beta \in \mathscr{R}$ such that $\left|\alpha^{\prime}\right|<1$, $\left|\beta^{\prime}\right|<1, \beta>\alpha>1$, and $\left|\beta^{\prime}\right|<\left|\alpha^{\prime}\right|$. If $\eta_{\alpha} \eta_{\beta}>0$ and $\left|\alpha^{\prime}+1\right| \leq 1$, then $\beta \geq \alpha+1$.

LeMMA 2.11 (Lemma 6.5 of [3]). Let $\alpha, \beta, \gamma \in \mathscr{R}$ such that $\left|\alpha^{\prime}\right|<1$, $\left|\beta^{\prime}\right|<1,\left|\gamma^{\prime}\right|<1,\left|\alpha^{\prime}-1\right|>1,\left|\beta^{\prime}-1\right|>1,\left|\gamma^{\prime}-1\right|>1,\left|\beta^{\prime}+1\right| \leq 1$, $\eta_{\alpha} \eta_{\beta}<0, \eta_{\beta} \eta_{\gamma}>0$. If $\left|\beta^{\prime}-\alpha^{\prime}\right|>1$ and $\left|\beta^{\prime}-\gamma^{\prime}+1\right|>1$, then either $\left|\alpha^{\prime}-\beta^{\prime}\right| \leq 1$ or $\left|\alpha^{\prime}-\beta^{\prime}+\gamma^{\prime}-1\right| \leq 1$.

3. The main results. We are now able to use the lemmas of $\$ 2$ to prove our main results. We first prove an extension of Lemma 2.11.

THEOREM 3.1. If $\alpha, \beta, \gamma \in \mathscr{R},\left|\alpha^{\prime}\right|<1,\left|\beta^{\prime}\right|<1,\left|\gamma^{\prime}\right|<1,\left|\alpha^{\prime}-1\right|>1$, $\left|\beta^{\prime}-1\right|>1,\left|\gamma^{\prime}-1\right|>1,\left|\beta^{\prime}+1\right| \leq 1, \eta_{\alpha} \eta_{\beta}<0, \eta_{\beta} \eta_{\gamma}>0$, and $\mid \beta^{\prime}-$ $\gamma^{\prime} \mid>1$, then either $\left|\alpha^{\prime}-\beta^{\prime}\right|<1$ or $\left|\alpha^{\prime}-\gamma^{\prime}\right| \leq 1$, where $\lambda=\beta-\gamma+1$. 
Proof. If $\left|\lambda^{\prime}\right|>1$, the result follows from Lemma 2.11. Note that since $\eta_{\alpha} \eta_{\beta}<0$, we cannot have $\left|\alpha^{\prime}-\beta^{\prime}\right|=1$, for this would imply that $\alpha=\beta \pm 1$ and $\eta_{\alpha}=\eta_{\beta}$. Similarly $\left|\alpha^{\prime}-\gamma^{\prime}\right| \neq 1$. If $\left|\lambda^{\prime}\right|=1$, then $\beta=\gamma$ or $\beta=\gamma-2$. Since $\left|\beta^{\prime}-\gamma^{\prime}\right|>0$ and $\left|\beta^{\prime}\right|<1,\left|\gamma^{\prime}\right|<1$, neither of these is possible.

If $\left|\lambda^{\prime}\right|<1$, we will consider two cases; however, we first notice that $\left|\gamma^{\prime}+1\right|>1$ by Lemma 2.8 and $\eta_{\gamma} \eta_{\lambda}=\eta_{\gamma}\left(\eta_{\beta}-\eta_{\gamma}\right)=\left|\eta_{\gamma}\right|\left(\left|\eta_{\beta}\right|-\left|\eta_{\gamma}\right|\right)<0$ by Lemma 2.3. Also $\kappa(\lambda)<1$ by Corollary 2.6 .1 .

Case $1(\kappa(\alpha)<1)$. In this case we see from Lemma 2.5 that $\left|\alpha^{\prime}-\lambda^{\prime}\right|$ $\leq 1$.

Case $2(\kappa(\alpha) \geq 1)$. In this case we have $\left|\alpha^{\prime}+1\right|<1$ by Corollary 2.4.1. Suppose $\left|\alpha^{\prime}-\gamma^{\prime}\right|>1$ and $\left|\alpha^{\prime}-\lambda^{\prime}\right|>1$. Since $\left|\beta^{\prime}-\gamma^{\prime}\right|>1$, we have $\left|\lambda^{\prime}-1\right|>1$; thus, $\left|\lambda^{\prime}+1\right|>1$ by Lemma 2.8 . If $\rho=\alpha-\lambda+1$ and $\left|\rho^{\prime}\right|>1$, then either $\left|\gamma^{\prime}-\rho^{\prime}\right| \leq 1$ or $\left|\gamma^{\prime}-\alpha^{\prime}\right| \leq 1$ by Lemma 2.11. Since $\gamma-\rho=\beta-\alpha$, we get $\left|\beta^{\prime}-\alpha^{\prime}\right|<1$. If $\left|\rho^{\prime}\right|=1$, then $\alpha=\lambda$ or $\alpha=\lambda-2$ and, as above, neither of these is possible. If $\left|\rho^{\prime}\right|<1$, then $\kappa(\rho)<1$ and also $\eta_{\rho} \eta_{\gamma}>0$ (Corollary 2.6.1 and Lemma 2.3). Since $\kappa(\gamma)<1$ by Corollary 2.4.1, we get $\left|\gamma^{\prime}-\rho^{\prime}\right| \leq 1$ by Lemma 2.5.

We are now able to show that if $\theta_{4}<\theta_{2}+1$, then $\theta_{4}+1=\theta_{2}+\theta_{3}$.

THEOREM 3.2. If $\chi<\theta+1$, then $\eta_{\theta} \eta_{\omega}<0,\left|\chi^{\prime}+1\right|<1, \kappa(\theta)<1$, $\kappa(\omega)<1$, and $\chi+1=\theta+\omega$.

Proof. We first note that $\left|\theta^{\prime}\right|<1,\left|\omega^{\prime}\right|<1,\left|\chi^{\prime}\right|<1$, and $\left|\theta^{\prime}-1\right|>1$, $\left|\omega^{\prime}-1\right|>1,\left|\chi^{\prime}-1\right|>1$. Also, if $\rho_{1}, \rho_{2} \in\{\theta, \omega, \chi\}$ and $\rho_{1} \neq \rho_{2}$, then $\left|\rho_{1}^{\prime}-\rho_{2}^{\prime}\right|>1$ by Lemma 2.1 .

Case $1\left(\eta_{\theta} \eta_{\omega}>0\right)$. By Lemma 2.4 we must have $\eta_{\theta} \eta_{\chi}<0$. Further, by Lemma 2.10, we must also have $\left|\theta^{\prime}+1\right|>1$. By Theorem 3.1, we get $\left|\rho^{\prime}\right| \leq 1$, where $\rho=\chi-\omega+\theta-1$. Now $0<\rho<\theta$; thus, $\rho=1$ and $\chi=\omega-\theta+2$. Since $\omega-\theta+1=\chi-1$, we have $\left|\omega^{\prime}-\theta^{\prime}+1\right|>1$; consequently, $\left|\chi^{\prime}\right|=\left|\omega^{\prime}-\theta^{\prime}+2\right|>1$ by Lemma 2.2. It follows that we must have

Case $2\left(\eta_{\theta} \eta_{\omega}<0\right)$. Here we have $\eta_{\chi} \eta_{\theta}>0$ or $\eta_{\chi} \eta_{\omega}>0$. In either case, by Lemma 2.10 we get $\left|\chi^{\prime}+1\right|<1$. If $\eta_{x} \eta_{\omega}>0$, then $\left|\omega^{\prime}+1\right|>1$ by Lemma 2.8 and $\kappa(\omega)<1$ by Corollary 2.4.1. Also, by Theorem 3.1 $\left|\rho^{\prime}\right| \leq 1$, where $\rho=\theta-\chi+\omega-1$. Since $-1<\rho<\theta$, we get $\rho=0$ or 1 . 
As before, we cannot have $\rho=1$; hence, $\rho=0$ and $\chi+1=\theta+\omega$. Since $\theta=\chi-\omega+1$, we get $\kappa(\theta)<1$ from Corollary 2.6.1. Similarly, if $\eta_{\chi} \eta_{\theta}$ $>0$, then $\kappa(\theta)<1, \kappa(\omega)<1$, and $\chi+1=\theta+\omega$.

By using the remarks at the beginning of $\S 2$, we can extend this result to show that if

$$
\theta_{n+3}<\theta_{n+1}+\theta_{n}
$$

in (1.1), then

$$
\theta_{n+3}+\theta_{n}=\theta_{n+1}+\theta_{n+2} .
$$

We can also improve two of the results of Theorem 3.2 in

LeMma 3.3. If $\chi<\theta+1$, then $\left|\theta^{\prime}+1\right|>1$ and $\left|\omega^{\prime}+1\right|>1$.

Proof. If $\left|\theta^{\prime}+1\right| \leq 1$, then $\zeta_{\theta} \leq 0$ and

$$
-2 \zeta_{\theta} \geq \zeta_{\theta}^{2}+\eta_{\theta}^{2}>\zeta_{\omega}^{2}+\eta_{\omega}^{2}>2 \zeta_{\omega} \quad\left(\left|\omega^{\prime}-1\right|>1\right) .
$$

It follows that $\zeta_{\theta}+\zeta_{\omega}<0$ and, as a consequence, $\left|\chi^{\prime}\right|=\left|\theta^{\prime}+\omega^{\prime}-1\right|>1$, which is impossible.

If $\left|\omega^{\prime}+1\right| \leq 1$, then $\zeta_{\omega} \leq 0$ and

$$
\left|\eta_{\omega}\right| \leq \sqrt{1-\left(1-\left|\zeta_{\omega}\right|\right)^{2}}
$$

Since

$$
2\left|\zeta_{\omega}\right|=\zeta_{\omega}^{2}+1-\left(1-\left|\zeta_{\omega}\right|\right)^{2}
$$

we get

$$
2\left|\zeta_{\omega}\right| \geq 1-\left(1-\left|\zeta_{\omega}\right|\right)^{2}>\left|\eta_{\omega}\right|\left(1-\left(1-\left|\zeta_{\omega}\right|\right)^{2}\right)
$$

Also, since

$$
\left(\left|\eta_{\theta}\right| \sqrt{2\left|\zeta_{\omega}\right|}-\sqrt{1-\left(1-\left|\zeta_{\omega}\right|\right)^{2}}\right)^{2} \geq 0
$$

we see, using (3.2), that

$$
\left(1-\eta_{\theta}^{2}\right) \zeta_{\omega}^{2} \leq\left(\sqrt{2\left|\zeta_{\omega}\right|}-\left|\eta_{\theta}\right| \sqrt{1-\left(1-\left|\zeta_{\omega}\right|\right)^{2}}\right)^{2}
$$

and

$$
\left|\zeta_{\omega}\right| \sqrt{1-\eta_{\theta}^{2}}+\left|\eta_{\theta}\right| \sqrt{1-\left(1-\left|\zeta_{\omega}\right|\right)^{2}} \leq \sqrt{2\left|\zeta_{\omega}\right|}
$$

by (3.3). Now $\zeta_{\theta}<\sqrt{1-\eta_{\theta}^{2}}$; hence from (3.1) we get

$$
\zeta_{\theta}\left|\zeta_{\omega}\right|+\left|\eta_{\omega}\right|\left|\eta_{\theta}\right|-\left|\zeta_{\omega}\right|<\sqrt{2\left|\zeta_{\omega}\right|}-\left|\zeta_{\omega}\right| \leq 1 / 2 .
$$


Since $\zeta_{\omega} \leq 0$ and $\eta_{\omega} \eta_{\theta}<0$, we find that

$$
-2 \zeta_{\omega}+2 \zeta_{\omega} \zeta_{\theta}+2 \eta_{\omega} \eta_{\theta}>-1 \text {. }
$$

But

$$
\begin{aligned}
\left|\chi^{\prime}\right|^{2}-\left|\omega^{\prime}\right|^{2} & =\left|\theta^{\prime}+\omega^{\prime}-1\right|^{2}-\left|\omega^{\prime}\right|^{2} \\
& =\left|\theta^{\prime}-1\right|^{2}-2 \zeta_{\omega}+2 \zeta_{\omega} \zeta_{\theta}+2 \eta_{\theta} \eta_{\omega} ;
\end{aligned}
$$

thus, since $\left|\theta^{\prime}-1\right|>1$, we have $\left|\chi^{\prime}\right|>\left|\omega^{\prime}\right|$ when $\left|\omega^{\prime}+1\right| \leq 1$ and this is impossible.

We will also need to make use of the following result and its corollaries.

THEOREM 3.4. If $\chi<\theta+1$ and there exists some $\rho \in \mathscr{R}$ such that $\rho \notin\{\theta, \omega, \chi\},\left|\rho^{\prime}\right|<1,\left|\rho^{\prime}-1\right|>1$, then $\left|\rho^{\prime}-\psi^{\prime}\right|<1$ for some $\psi \in$ $\{\theta, \omega, \chi\}$.

Proof. Suppose that there exists some $\rho \in \mathscr{R}$ such that $\rho \notin\{\theta, \omega, \chi\}$, $\left|\rho^{\prime}\right|<1,\left|\rho^{\prime}-1\right|>1$, and $\left|\rho^{\prime}-\psi^{\prime}\right| \geq 1$ for each $\psi \in\{\theta, \omega, \chi\}$. We first note that if $\left|\rho^{\prime}-\psi^{\prime}\right|=1$, then $\rho=\psi+1$. If $\rho=\psi-1$, then $0<\rho<\theta$, which contradicts the definition of $\theta$. If $\rho=\psi+1$, then $\left|\rho^{\prime}-1\right|=\left|\psi^{\prime}\right|<$ 1 , which is also impossible. Thus, $\left|\rho^{\prime}-\psi^{\prime}\right|>1$ for all $\psi \in\{\theta, \omega, \chi\}$. Since $\eta_{\theta} \eta_{\omega}<0,\left|\theta^{\prime}+1\right|>1,\left|\omega^{\prime}+1\right|>1$, we must have $\left|\rho^{\prime}+1\right|<1$ (Lemma 2.8). Put $\alpha$ equal to that one of $\theta$ or $\omega$ such that $\eta_{\alpha} \eta_{\rho}<0$ and let $\beta$ be the other one. We have $\alpha+\beta=\theta+\omega=\chi+1$. Further, $\left|\rho^{\prime}-\alpha^{\prime}\right|$ $>1$ and $\left|\alpha^{\prime}+1\right|>1$; thus, by Theorem 3.1, we get $\left|\beta^{\prime}-\lambda^{\prime}\right| \leq 1$, where $\lambda=\rho-\alpha+1$. Since $\beta-\lambda=\beta-\rho+\alpha-1=\chi-\rho$, this is impossible.

COROllary 3.4.1. If $\chi<\theta+1$ and there exists $\rho \in \mathscr{R}$ such that $\rho \in\{\theta, \omega, \chi\},\left|\rho^{\prime}\right|<1$, and $|\rho|<\theta+1$, then $\rho=0$.

Proof. Since $\left|-\rho^{\prime}\right|=\left|\rho^{\prime}\right|$, we may assume with no loss of generality that if $\rho \neq 0$, then $\rho>0$. Since $\left|\rho^{\prime}\right|<1$, we must have $\theta<\rho<\theta+1$. Thus, by Lemma $2.1,\left|\rho^{\prime}-\psi^{\prime}\right| \geq 1$ for all $\psi \in\{\theta, \omega, \chi\}$, which is impossible by the theorem.

CoRollary 3.4.2. If $\chi<\theta+1$, there does not exist any $\rho \in \mathscr{R}$ such that $\left|\rho^{\prime}\right|<1$ and $\chi<\rho<\chi+1$. 
Proof. Suppose such a $\rho$ does exist. If $\left|\rho^{\prime}-1\right|<1$, then, since $|\rho-1|<\theta+1$, we can only have $\rho-1 \in\{\theta, \omega, \chi\}$ by the previous result. Since $\rho \neq \chi+1,\left|\theta^{\prime}+1\right|>1,\left|\omega^{\prime}+1\right|>1$, we must have $\left|\rho^{\prime}-1\right|$ $>1$ and, as a consequence, $\left|\rho^{\prime}-\psi^{\prime}\right|<1$ for some $\psi \in\{\theta, \omega, \chi\}$. Since $0<\rho-\chi<\chi+1-\chi \leq \omega$, we find by the previous corollary that $\rho-$ $\psi=\theta$. If $\psi=\omega$ or $\chi$, then $\rho \geq \chi+1$; thus, $\psi=\theta$ and $\rho=2 \theta$. If $\rho=2 \theta$, then $\left|\omega^{\prime}\right|<\left|\theta^{\prime}\right|<1 / 2$ and $\left|\omega^{\prime}-\theta^{\prime}\right|<1$, which is impossible.

Let $\rho=\theta_{5}$, the minimum adjacent to $\chi=\theta_{4}$. We can now show the following unconditional result concerning $\rho$.

THEOREM 3.5. $\rho \geq 1+\omega$ or $\theta_{n+5} \geq \theta_{n+3}+\theta_{n}$ in (1.1).

Proof. Suppose $\rho<1+\omega$ and let $\mathscr{R}^{*}=(1 / \theta) \mathscr{R}$. If $\theta^{*}=\omega / \theta$, $\omega^{*}=\chi / \theta, \chi^{*}=\rho / \theta$, then $\theta^{*}$ is the minimum adjacent to 1 in $\mathscr{R}^{*}, \omega^{*}$ is the minimum adjacent to $\theta^{*}$, and $\chi^{*}$ is the minimum adjacent to $\omega^{*}$. Since $\rho<1+\omega$, we have $\chi^{*}<(1+\omega) / \theta<\omega / \theta+1=\theta^{*}+1$. By Theorem 3.2, we have $\theta^{*}+\omega^{*}=\chi^{*}+1$ and

$$
\omega+\chi=\rho+\theta \text {. }
$$

If $\chi \geq \theta+1$, then $\rho \geq \omega+1$. If $\chi<\theta+1$, then $\rho \geq \chi+1>\omega+1$ by Corollary 3.4.2.

In fact, we actually get cases in which $\rho=1+\omega$. For example, consider $D=239, \delta^{3}=D, \mathscr{R}_{1}=\left\langle 1, \delta, \delta^{2}\right\rangle$. In $\mathscr{R}=\mathscr{R}_{312}$, we get

$$
\begin{aligned}
& \theta=\left(6+17 \delta+7 \delta^{2}\right) / 247, \\
& \omega=\left(74+45 \delta+4 \delta^{2}\right) / 247, \\
& \chi=\left(253+17 \delta+7 \delta^{2}\right) / 247=\theta+1, \\
& \rho=\left(321+45 \delta+4 \delta^{2}\right) / 247=\omega+1 .
\end{aligned}
$$

Note also that if $\mathscr{R}=\mathscr{R}_{313}$ here, we have $\theta=\left(191-3 \delta+7 \delta^{2}\right) / 332$, $\omega=\left(217+47 \delta+\delta^{2}\right) / 332, \chi=\left(76+44 \delta+8 \delta^{2}\right) / 332$. In this case $\chi<$ $\theta+1$ and $\chi=\theta+\omega-1$. Also, $\rho=\left(408+44 \delta+8 \delta^{2}\right) / 332=\chi+1$.

If we let $\mathscr{R}_{1}=\langle 1, \mu, \nu\rangle$, where $\{1, \mu, \nu\}$ is a basis of the algebraic integers of $\mathscr{K}$, then $\mathscr{R}_{1}$ is a reduced lattice and there exists an integer $p$ such that $\mathscr{R}_{p+1}=\mathscr{R}_{1}$. In this case $\varepsilon_{0}(>1)$, the fundamental unit of $\mathscr{K}$, is given by the formula

$$
\varepsilon_{0}=\theta_{p+1}=\prod_{i=1}^{p} \theta_{g}^{(i)}
$$


The value $p$ is called the period of Voronoi's continued fraction algorithm for finding $\varepsilon_{0}$. By using the reasoning similar to that of Pen and Skubenko [2], we can prove

THEOREM 3.6. If $p$ is the period of Voronoi's continued fraction algorithm for finding $\varepsilon_{0}$, then $\varepsilon_{0}>\tau^{p / 2}$, where $\tau=(1+\sqrt{5}) / 2$.

$$
\begin{aligned}
\text { Proof. If } \mathscr{R}= & \mathscr{R}_{i} \text {, then } \rho \geq \omega+1 \text { and } \\
& \boldsymbol{\theta}_{g}^{(i)} \boldsymbol{\theta}_{g}^{(i+1)} \theta_{g}^{(i+2)} \theta_{g}^{(i+3)} \geq 1+\boldsymbol{\theta}_{g}^{(i)} \theta_{g}^{(i+1)} .
\end{aligned}
$$

Since $\mathscr{R}_{p+1}=\mathscr{R}_{1}, \mathscr{R}_{p+2}=\mathscr{R}_{2}, \mathscr{R}_{p+3}=\mathscr{R}_{3}$, we get $\theta_{g}^{(p+1)}=\theta_{g}^{(1)}, \theta_{g}^{(p+2)}$ $=\theta_{g}^{(2)}, \theta_{g}^{(p+3)}=\theta_{g}^{(3)}$; thus, we get

$$
\begin{aligned}
\varepsilon_{0}^{4} & =\left(\prod_{i=1}^{p} \theta_{g}^{(i)}\right)^{4}=\prod_{i=1}^{p} \theta_{g}^{(i)} \theta_{g}^{(i+1)} \theta_{g}^{(i+2)} \theta_{g}^{(i+3)} \\
& \geq \prod_{i=1}^{p}\left(1+\theta_{g}^{(i)} \theta_{g}^{(i+1)}\right) \\
& \geq \prod_{i=1}^{p}\left(1+\left(\prod_{i=1}^{p} \theta_{g}^{(i)} \theta_{g}^{(i+1)}\right)^{1 / p}\right)^{p} \\
& =\left(1+\varepsilon_{0}^{2 / p}\right)^{p} .
\end{aligned}
$$

If we put $\eta=\varepsilon_{0}^{2 / p}>1$, then $\eta^{2} \geq \eta+1$. It follows that $\varepsilon_{0}^{2 / p}>\tau$.

Thus, if $R$ is the regulator of $\mathscr{K}$, we have $R>p(\log \tau) / 2$.

4. Further results. In this section we will obtain some results on the spacing of the first few minima of $\mathscr{R}$. We first require the following technical lemma.

LEMMA 4.1. If $\chi<\theta+1$, then

(i) $\left|\theta^{\prime}\right|,\left|\omega^{\prime}\right|>1 / 2$;

(ii) $\left|2 \omega^{\prime}+\chi^{\prime}\right|>\left|\omega^{\prime}\right|,\left|2 \theta^{\prime}+\chi^{\prime}\right|>\left|\theta^{\prime}\right|,\left|2 \theta^{\prime}+\omega^{\prime}\right|>\left|\theta^{\prime}\right|$;

(iii) $\left|\theta^{\prime}+\chi^{\prime}\right|>\left|\chi^{\prime}\right|$;

(iv) $\left|2 \chi^{\prime}+\theta^{\prime}\right|>\left|\chi^{\prime}\right|$.

Proof. (i) The method of proof of (i) is given in the proof of Corollary 3.4.2.

(ii) Since $\left|\omega^{\prime}\right|>\left|\chi^{\prime}\right|$, we have

$$
\left|2 \omega^{\prime}+\chi^{\prime}\right| \geq 2\left|\omega^{\prime}\right|-\left|\chi^{\prime}\right|>\left|\omega^{\prime}\right| .
$$

Similarly, $\left|2 \theta^{\prime}+\chi^{\prime}\right|>\left|\theta^{\prime}\right|$ and $\left|2 \theta^{\prime}+\omega^{\prime}\right|>\left|\theta^{\prime}\right|$. 
(iii) We note that

$$
2 \zeta_{\chi} \zeta_{\theta}+2 \eta_{\chi} \eta_{\theta}=\left|\chi^{\prime}+1\right|^{2}-\left|\chi^{\prime}+1-\theta^{\prime}\right|^{2}+\left|\theta^{\prime}-1\right|^{2}-1
$$

Since $\omega=\chi+1-\theta$, we get

$$
\left|\theta^{\prime}+\chi^{\prime}\right|^{2}=\left|\theta^{\prime}\right|^{2}+\left|\chi^{\prime}\right|^{2}+\left|\chi^{\prime}+1\right|^{2}-\left|\omega^{\prime}\right|^{2}+\left|\theta^{\prime}-1\right|^{2}-1
$$

Since $\left|\theta^{\prime}\right|>\left|\omega^{\prime}\right|$ and $\left|\theta^{\prime}-1\right|>1$, we have

$$
\left|\theta^{\prime}+\chi^{\prime}\right|>\left|\chi^{\prime}\right| \text {. }
$$

(iv) From (4.1) we get

$$
\begin{aligned}
\left|2 \chi^{\prime}+\theta^{\prime}\right|^{2}-\left|\chi^{\prime}\right|^{2}= & \left|\chi^{\prime}\right|^{2}+2\left|\chi^{\prime}\right|^{2}+2\left|\chi^{\prime}+1\right|^{2}-\left|\omega^{\prime}\right|^{2} \\
& +\left|\theta^{\prime}\right|^{2}-\left|\omega^{\prime}\right|^{2}+2\left|\theta^{\prime}-1\right|^{2}-2 .
\end{aligned}
$$

Since

$$
\begin{aligned}
\left|\chi^{\prime}\right|^{2}+\left|\chi^{\prime}+1\right|^{2} & \geq \zeta_{\chi}^{2}+\left(\zeta_{\chi}+1\right)^{2} \\
& =\frac{1}{2}\left(4 \zeta_{\chi}^{2}+4 \zeta_{\chi}+1\right)+\frac{1}{2} \geq \frac{1}{2}
\end{aligned}
$$

we get

$$
\left|2 \chi^{\prime}+\theta^{\prime}\right|-\left|\chi^{\prime}\right|>0 \text {. }
$$

We are now able to find possible candidates for further minima when $\chi<\theta+1$.

LEMMA 4.2. If $\chi<\theta+1, \chi+1<\rho<\chi+2$, and $\left|\rho^{\prime}\right|<1$, then $\rho \in\{\chi+\theta, \chi+\omega, 2 \chi\}$.

Proof. Since $\chi<\rho-1<\chi+1$, we cannot have $\left|\rho^{\prime}-1\right| \leq 1$, by Corollary 3.4.2. Since $\left|\rho^{\prime}-1\right|>1$, by Theorem 3.4, we must have some $\psi \in\{\theta, \omega, \chi\}$ such that $\left|\rho^{\prime}-\chi^{\prime}\right|<1$. If $\psi=\theta$, then

$$
\omega=\chi+1-\theta<\rho-\psi<\chi+2-\theta=\omega+1<\chi+1 \text {; }
$$

hence, $\rho-\theta=\chi$ by Corollary 3.4.1 and 3.4.2. If $\psi=\omega$, then $\theta<\rho-\chi$ $<\theta+1$. By Corollary 3.4.1, we can only have $\rho=2 \omega$, which is impossible by Lemma 4.1 , or $\rho=\omega+\chi$. If $\psi=\chi$, then $1<\rho-\psi<1+\theta$ and $\rho-\chi \in\{\theta, \omega, \chi\}$.

COROLlaRY 4.2.1. If $\rho$ satisfies the conditions of the lemma and $\rho$ is also a minimum of $\mathscr{R}$, then $\rho=\chi+\omega$.

Proof. If $\rho=2 \chi$ or $\rho=\theta+\chi$, then $\left|\rho^{\prime}\right|>\left|\chi^{\prime}\right|$, which is not possible. 
LEMMA 4.3. If $\chi<\theta+1, \chi+2<\rho<\chi+3$, and $\left|\rho^{\prime}\right|<1$, then

$$
\begin{array}{r}
\rho \in\{\theta+\chi, \omega+\chi, 2 \chi, \chi+\theta+1, \chi+\omega+1, \chi+2 \theta, \\
\chi+2 \omega, 2 \chi+1,2 \chi+\theta, 2 \chi+\omega, 3 \chi\} .
\end{array}
$$

Proof. Since $\chi+1<\rho-1<\chi+2$, we see by Lemma 4.2 that if $\left|\rho^{\prime}-1\right|<1$, then $\rho=\chi+\theta+1, \chi+\omega+1,2 \chi+1$. If $\left|\rho^{\prime}-1\right| \geq 1$, then $\left|\rho^{\prime}-\psi^{\prime}\right|<1$ for some $\psi \in\{\theta, \omega, \chi\}$. If $\psi=\theta$, then

$$
\chi<\omega+1<\chi+2-\theta<\rho-\psi<\chi+3-\theta=\omega+2<\chi+2 .
$$

Thus, $\rho-\theta \in\{\chi+1, \chi+\theta, \chi+\omega, 2 \chi\}$. (Note that $\theta+\omega+\chi=2 \chi$ +1 .) If $\psi=\omega$, then $\chi<\rho-\chi<\chi+2$ and $\rho-\omega \in\{\chi+1, \chi+\theta$, $\chi+\omega, 2 \chi\}$. If $\psi=\chi$, then $2<\rho<\chi+2$ and $\rho-\chi \in\{\theta, \chi, \omega, \chi+1$, $\chi+\theta, \chi+\omega, 2 \chi\}$.

COROLlaRy 4.3.1. If $\rho$ satisfies the conditions of the lemma and $\rho$ is a minimum of $\mathscr{R}$, then

$$
\rho \in\{\omega+\chi, \omega+\chi+1,2 \chi+1,2 \chi+\omega\} .
$$

Proof. We have $2\left|\chi^{\prime}\right|, 3\left|\chi^{\prime}\right|>\left|\chi^{\prime}\right|$; the other possibilities are ruled out by Lemma 4.1.

THEOREM 4.4. If $\chi<\theta+1$, there does not exist a set of minima $\left\{\mu_{1}, \mu_{2}, \mu_{3}, \mu_{4}\right\}$ of $\mathscr{R}$ such that

$$
\chi+1 \leq \mu_{1}<\mu_{2}<\mu_{3}<\mu_{4}<\chi+3 .
$$

Proof. Put $\mathscr{R}^{*}=\left(1 / \mu_{1}\right) \mathscr{R}, \theta^{*}=\mu_{2} / \mu_{1}, \omega^{*}=\mu_{3} / \mu_{1}, \chi^{*}=\mu_{4} / \mu_{1}$. Since $\chi^{*}<(\chi+3) /(\chi+1)<1+\theta^{*}$, we must have

$$
\mu_{4}+\mu_{1}=\mu_{2}+\mu_{4} \quad \text { (Theorem 3.2), }
$$

and $\mu_{1}, \mu_{2}, \mu_{3}, \mu_{4} \in\{\chi+1, \chi+\omega, \chi+\omega+1,2 \chi+1,2 \chi+\omega\}$ by Corollaries 4.2.1 and 4.3.1. If $\mu_{1} \neq \chi+1$, then (4.2) cannot hold. If $\mu_{1}=\chi+1$ and $\mu_{2} \neq \chi+\omega+1$, then (4.2) again cannot hold. Thus, we must have $\omega_{1}=\chi+1$ and $\mu_{2}=\chi+\omega+1$. It follows that $\mu_{2}-\mu_{1}=\omega$ -1 and we can only have $\mu_{3}=2 \chi+1, \mu_{4}=2 \chi+\omega$.

Since $\chi+1$ is a minimum, we have $\left|\chi^{\prime}+1\right|<\left|\chi^{\prime}\right|$, and therefore $\zeta_{\chi}<-1 / 2$. Since $\zeta_{\omega}<1 / 2$, we get $2 \zeta_{\chi}+\zeta_{\omega}<-1 / 2$ and $\left|2 \chi^{\prime}+\omega^{\prime}+1\right|$ $<\left|\omega^{\prime}+\chi^{\prime}\right|$. Thus, if $\mu_{5}$ is the minimum adjacent to $\mu_{4}=2 \chi+\omega$, then $\mu_{5} \leq 2 \chi+\omega+1$. Since $\rho^{*}=\mu_{5} / \mu_{1}$, the minimum adjacent to $\chi^{*}$ in $\mathscr{R}^{*}$, must satisfy $\rho^{*} \geq \chi^{*}+1$, we get $\mu_{5} \geq \mu_{4}+\mu_{1}=3 \chi+\omega+1>2 \chi+\omega$ +1 , a contradiction. 
Corollary 4.4.1. If $\theta_{1}=1$ in (2.1), then $\theta_{8}>4$.

Proof. If $\theta_{4} \geq \theta_{1}+1$, put $\mathscr{R}^{*}=\left(1 / \theta_{4}\right) \mathscr{R}, \theta^{*}=\theta_{5} / \theta_{4}, \omega^{*}=\theta_{6} / \theta_{4}$, $\chi^{*}=\theta_{7} / \theta_{4}, \rho^{*}=\theta_{8} / \theta_{4}$. By Theorem 3.5, we have $\rho^{*} \geq \omega^{*}+1$; hence, $\theta_{8}=\theta_{4} \rho^{*} \geq\left(\theta_{1}+1\right)\left(\omega^{*}+1\right)>4$. If $\theta_{4}<\theta_{1}+1$, then $\theta_{8}>\theta_{5}+3>4$ by the theorem.

It follows from Corollary 4.4 .1 that in $\mathscr{R}_{i}$ we have

$$
\prod_{j=0}^{6} \theta_{g}^{(i+j)}>4
$$

hence, from (2.1), we get

$$
\theta_{n}>4^{[(n-1) / 7]}
$$

\section{REFERENCES}

[1] B. N. Delone and D. K. Faddeev, The Theory of Irrationalities of the Third Degree, Amer. Math. Soc., Providence, RI, 1964.

[2] A. S. Pen and B. F. Skubenko, Estimation from above of the period of a quadratic irrationality, Mat. Zametki, 5 (1969), 413-418.

[3] H. C. Williams and G. W. Dueck, An analogue of the nearest integer continued fraction for certain cubic irrationalities, Math. Comp., 42 (1984), 683-705.

Received July 6, 1984. Research supported by NSERC of Canada Grant \#A7649 and by the I. W. Killam Foundation.

UNIVERSITY OF MANITOBA

WinNIPEg, MANITOBa

Canada R3T 2N2 


\section{PACIFIC JOURNAL OF MATHEMATICS EDITORS}

\author{
V. S. VARADARAJAN \\ (Managing Editor) \\ University of California \\ Los Angeles, CA 90024 \\ Herbert Clemens \\ University of Utah \\ Salt Lake City, UT 84112 \\ R. FINN \\ Stanford University \\ Stanford, CA 94305
}

\author{
HERMANN FLASCHKA \\ University of Arizona \\ Tucson, AZ 85721 \\ RAMESH A. GANGOLLI \\ University of Washington \\ Seattle, WA 98195 \\ VAUGHAN F. R. JONES \\ University of California \\ Berkeley, CA 94720 \\ ROBION KIRBY \\ University of California \\ Berkeley, CA 94720
}

C. C. MOORE

University of California

Berkeley, CA 94720

H. SAMELSON

Stanford University

Stanford, CA 94305

HAROLD STARK

University of California, San Diego La Jolla, CA 92093

\section{ASSOCIATE EDITORS}
R. ARENS
E. F. BECKENBACH (1906-1982)
B. H. NEUMANN
F. WOLF
K. YosHIDA

\section{SUPPORTING INSTITUTIONS}

\begin{abstract}
UNIVERSITY OF ARIZONA
UNIVERSITY OF BRITISH COLUMBIA

CALIFORNIA INSTITUTE OF TECHNOLOGY

UNIVERSITY OF CALIFORNIA

MONTANA STATE UNIVERSITY

UNIVERSITY OF NEVADA, RENO

NEW MEXICO STATE UNIVERSITY OREGON STATE UNIVERSITY
\end{abstract}

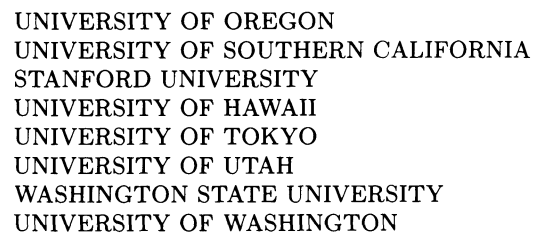

The Supporting Institutions listed above contribute to the cost of publication of this Journal, but they are not owners or publishers and have no responsibility for its content or policies.

Mathematical papers intended for publication in the Pacific Journal of Mathematics should be in typed form or offset-reproduced (not dittoed), double spaced with large margins. Please do not use built up fractions in the text of the manuscript. However, you may use them in the displayed equations. Underline Greek letters in red, German in green, and script in blue. The first paragraph must be capable of being used separately as a synopsis of the entire paper. In particular it should contain no bibliographic references. Please propose a heading for the odd numbered pages of less than 35 characters. Manuscripts, in triplicate, may be sent to any one of the editors. Please classify according to the scheme of Math. Reviews, Index to Vol. 39. Supply name and address of author to whom proofs should be sent. All other communications should be addressed to the managing editor, or Elaine Barth, University of California, Los Angeles, California 90024.

There are page-charges associated with articles appearing in the Pacific Journal of Mathematics. These charges are expected to be paid by the author's University, Government Agency or Company. If the author or authors do not have access to such Institutional support these charges are waived. Single authors will receive 50 free reprints; joint authors will receive a total of 100 free reprints. Additional copies may be obtained at cost in multiples of 50 .

The Pacific Journal of Mathematics is issued monthly as of January 1966. Regular subscription rate: $\$ 190.00$ a year (5 Vols., 10 issues). Special rate: $\$ 95.00$ a year to individual members of supporting institutions.

Subscriptions, orders for numbers issued in the last three calendar years, and changes of address should be sent to Pacific Journal of Mathematics, P.O. Box 969, Carmel Valley, CA 93924, U.S.A. Old back numbers obtainable from Kraus Periodicals Co., Route 100, Millwood, NY 10546.

The Pacific Journal of Mathematics at P.O. Box 969, Carmel Valley, CA 93924 (ISSN 0030-8730) publishes 5 volumes per year. Application to mail at Second-class postage rates is pending at Carmel Valley, California, and additional mailing offices. Postmaster: send address changes to Pacific Journal of Mathematics, P.O. Box 969, Carmel Valley, CA 93924.

PUBLISHED BY PACIFIC JOURNAL OF MATHEMATICS, A NON-PROFIT CORPORATION Copyright (C) 1986 by Pacific Journal of Mathematics 


\section{Pacific Journal of Mathematics}

Vol. 124, No. $2 \quad$ June, 1986

Philip Lee Bowers, Nonshrinkable "cell-like" decompositions of $s \ldots \ldots .257$

Aurelio Carboni and Ross Street, Order ideals in categories .......... 275

Leoni Dalla, Increasing paths on the one-skeleton of a convex compact set in

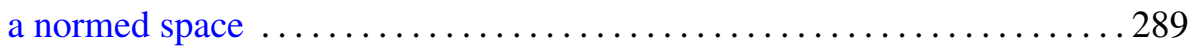

Jim Hoste, A polynomial invariant of knots and links ............... 295

Sheldon Katz, Tangents to a multiple plane curve ................... 321

Thomas George Lucas, Some results on Prüfer rings $\ldots \ldots \ldots \ldots \ldots \ldots 33$

Pham Anh Minh, Modular invariant theory and cohomology algebras of

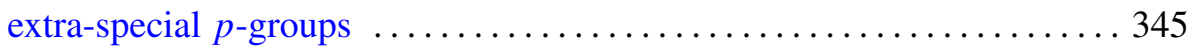

Ikuko Miyamoto, On inclusion relations for absolute Nörlund

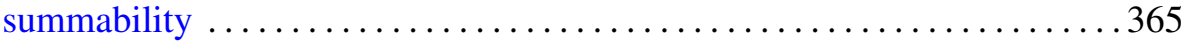

A. Papadopoulos, Geometric intersection functions and Hamiltonian flows on the space of measured foliations on a surface ............. 375

Richard Dean Resco, J. Toby Stafford and Robert Breckenridge

Warfield, Jr., Fully bounded $G$-rings $\ldots \ldots \ldots \ldots \ldots \ldots \ldots \ldots \ldots 4$

Haskell Paul Rosenthal, Functional Hilbertian sums .................417

Luen-Fai Tam, Regularity of capillary surfaces over domains with corners: borderline case ................................. 469

Hugh C. Williams, The spacing of the minima in certain cubic lattices .....483 